\title{
Tire Pressure and the Availability of Gasoline Monitoring Tools Based on IOT
}

\author{
Tandya Daviend Benaya Nugroho ${ }^{1}$, Albert Gunadhi ${ }^{1}$, Evelyn Raguindin ${ }^{2}$, \\ Hartono Pranjoto ${ }^{1, *}$
}

${ }^{1}$ Faculty of Electrical Engineering, Widya Mandala Catholic University, Jl. Kalijudan 37, Surabaya, East Java, 60144 Indonesia

${ }^{2}$ Electronics Engineering Department, Adamson University, 900 San Marcelino St, Ermita, Manila, 1000 Metro Manila, Philippines

\begin{abstract}
This final project discusses the design and manufacture of tire pressure and the availability of gasoline monitoring tools based on internet of things (IOT). Tire pressure monitoring tools are made because many of the motorcycle driver don't have a time to check tire pressure, and often experience a reduction in pressure to leak on the highway. While the gasoline monitoring tool is made so that driver can see the contents of the available motorcycle tank volume, and estimate the price paid when filling in the gas. Conventional methods of estimating gasoline prices often cause failure to refuel at gas stations, and make motorists complain about improper prices. Basically this tool works by means of a gasoline indicator and is connected to the ADC port of the NodeMCU microcontroller. As for tire pressure, the measurement is done by using a pressure sensor and additional $\mathrm{ADC}$, because the microcontroller only has one ADC port. Both of these tools work in the second way the microcontroller receives input data from the gasoline ADC indicator, and there is data processing in the microcontroller to connect the monitoring system via the internet.
\end{abstract}

Keywords: Analog to digital converter, internet connectivity, gasoline indicator, pressure indicator, NodeMCU microcontroller.

\section{Introduction}

Internet of things (IOT) is a technology that enables communication between devices over the internet [1-5]. This technology aims to expand the benefits of connected internet connectivity, enable control of communication with various hardware devices and from various data, to virtualize all real things through the internet network. The system used by the Internet of things is wireless or controls automatically without knowing distance.

The implementation of Internet of things itself usually depends on an individual or company agency in developing an application that is made, if the application is made to help monitor a room then implementing the Internet of things itself must follow the flow of programming diagrams regarding sensors in a room, how far so that the room can be controlled, and the internet network speed used. The fast network and speed of internet

\footnotetext{
*Corresponding author: pranjoto@ukwms.ac.id
} 
access can help implement the Internet of things to be more optimal, making it easier to control anything.

\section{IOT and hardware}

The internet of things is developing at a rapid pace, thanks in part to an explosion in the availability of small, inexpensive computing hardware. IOT prototyping kits and development boards combine microcontrollers and processors with wireless chips and other components in a pre-built, ready-to-program package. It come in nearly infinite configurations, from tiny battery-powered chips that chirp intermittently over Bluetooth to credit card-sized computers with USB power supplies and high-bandwidth WiFi radios. Whatever the needs of your project or product, there's sure to be a board that fits your exact requirements.

The ESP8266 is a low-cost WiFi microchip with full TCP/IP stack and microcontroller, and it is useful chip for IOT application. The chip first came to the attention of western makers in august 2014 with the ESP-01 module, made by a third-party manufacturer AIthinker. This small module allows microcontrollers to connect to a WiFI network and make simple TCP/IP connections using hayes-style commands.

\subsection{NodeMCU}

NodeMCU microcontroller is an ESP 8266 chip based module development microcontroller [6]. This microcontroller was made as a solution to the high cost of a microcontroller-based wireless system. Microcontrollers have the ability to provide WiFi connectivity facilities easily and the memory used is very large, which is $4 \mathrm{MB}$.

NodeMCU is an IoT platform consisting of hardware in the form of a system on chip ESP8266, and has a Wi-Fi feature that supports TCP/IP (internet protocol). The term NodeMCU basically refers to software used as a development kit. The NodeMCU is analogous to the Arduino board of ESP8266. This microcontroller has an $80 \mathrm{MHz}$ clock frequency, and has a flash memory of $4 \mathrm{MB}$, and can be used as an alternative to developing IoT devices.

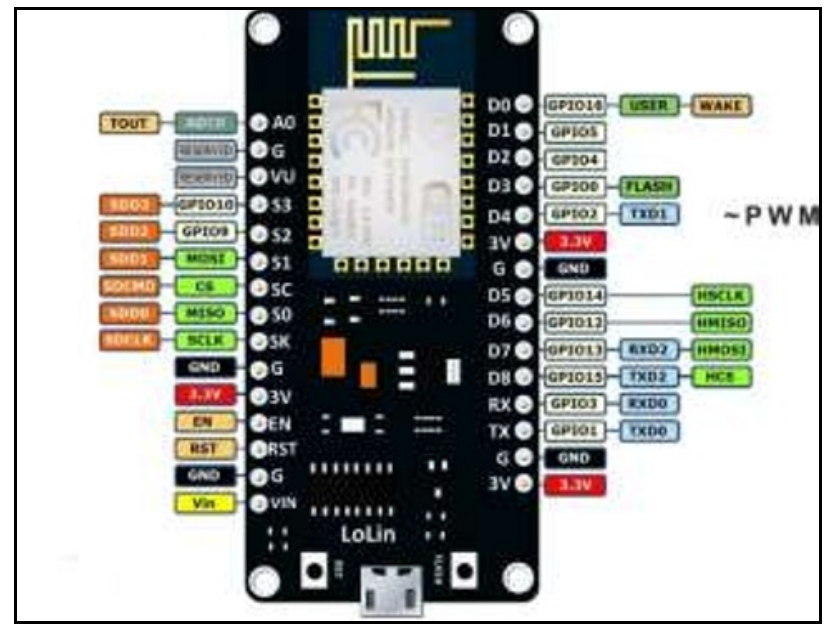

Fig. 1. NodeMCU \& its's ports. 


\subsection{Sensors of monitoring tools}

Every motorized vehicle has a device that can indicate the condition of the fuel tank, whether it is still fully charged or is almost gone. The tool is called fuel meter. Fuel meter, better known as a gasoline meter, generally consists of three basic parts, namely: a display in the form of a needle or digital, a variable resistor or potentiometer, and a buoy.

The working principle of the fuel meter is based on variations in resistance or changes in electrical resistance that occur due to fluctuating buoys in the gasoline tank following the surface level of the liquid [7]. When the gasoline is full, the buoy will rise, the resistance will be small, and the electric current will be large so the pointer will move upwards, on the contrary, when the gasoline is empty, the float drops, the resistance becomes large, and the electric current becomes small so the pointer drops. The fuel meter sensor (potentiometer) installed in ADC 10-bit at port 0 (A0) pin NodeMCU.

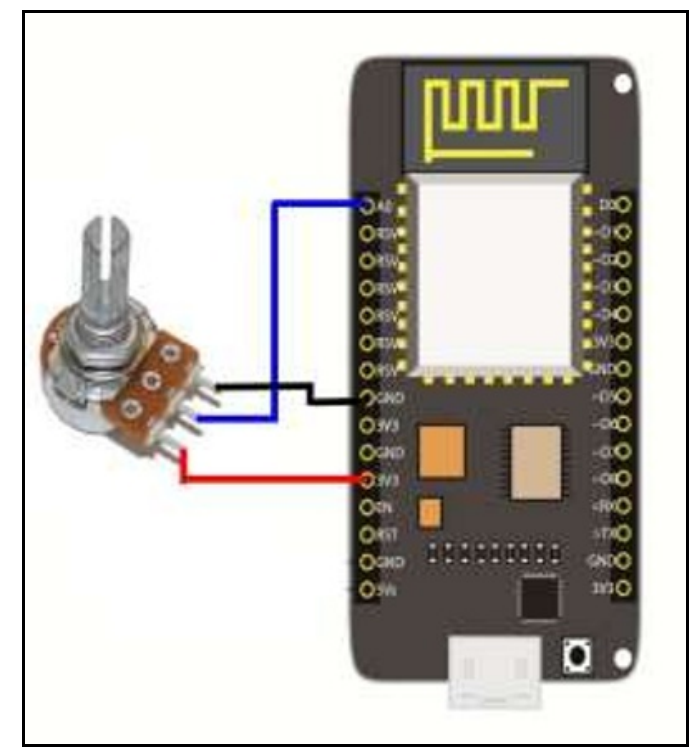

Fig. 2. Potentiometer and connected to port A0 NodeMCU.

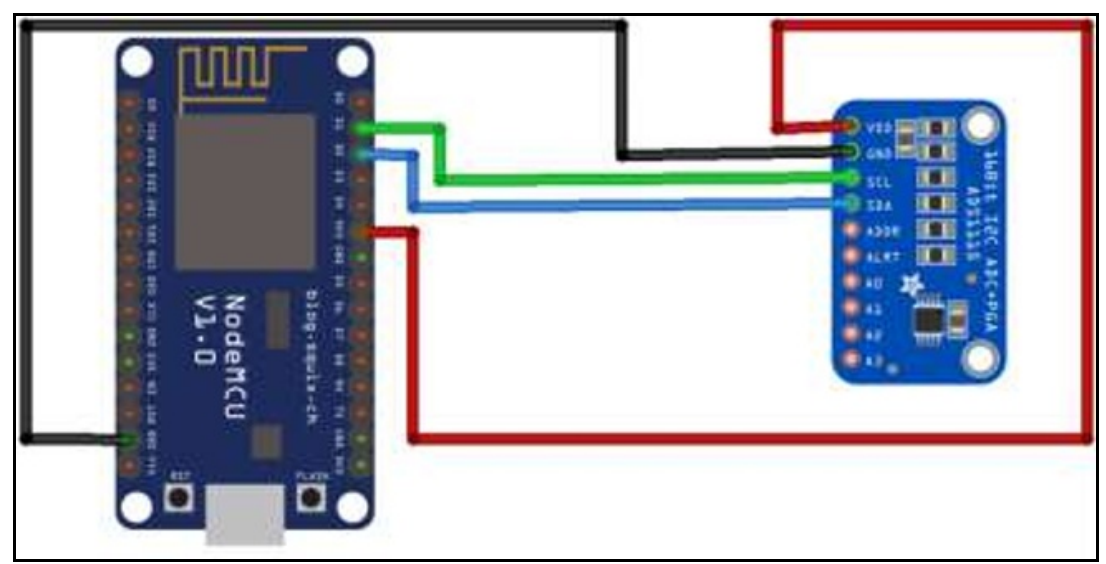

Fig. 3. ADS1115 connected to NodeMCU microcontroller. 
Meanwhile, pressure sensor sensor is a tool for measuring gas or liquid pressure $[8,9]$. Pressure is needed to stop the fluid from developing, and is usually expressed in force per unit area. Pressure sensors usually act as transducers which function to convert changes in mechanical pressure into electrical signals. Pressure sensors are used to measure other variables such as fluid or gas flow, speed, fluid height. ADS1115 is an external ADC device to read pressure sensor value that functions as a voltage reading with 16-bit resolution communication that has four channels $[10,11]$.

This device is easy to use by measuring various signals with a voltage range from $2 \mathrm{~V}$ to $5 \mathrm{~V}$. This module is very necessary if the ADC needs exceed the total number of NodeMCU microcontrollers, because in the NodeMCU microcontroller there is only one ADC.

\section{Programming}

Software design in this paper uses Arduino IDE software. Arduino IDE is compatible with microcontroller facilities such as: Arduino, Adafruit, ESP8266, ESP32. The Arduino IDE application is available for Linux, Windows, and Macintosh operating systems and can be used in 32 bit or 64 bit architectures.

\subsection{Microcontroller programming}

Microcontroller programming is the main program in the thesis, because it is used to regulate and control the entire work system design. The programming language for the microcontroller is C language which is packaged through Arduino IDE software with 9600 baudrate as data communication from the NodeMCU microcontroller and computer.

The purpose of this programming is to send input data from the server to the fuel meter driver so that it can detect directly as an indication of the volume of gasoline in the form of liters and its conversion into rupiah. There is also a pressure sensor that is useful as a monitor of tire pressure that will send data in the form of voltage to the microcontroller. Microcontroller programming is connected with the Blynk server and is connected to the Android smartphone operating system.

\subsection{Blynk}

Blynk is an open source or free android application that aims to control the NodeMCU microcontroller module via the internet. With the Blynk application, users will get convenience from prototypes to production at affordable prices. Blynk was made by engineers to simplify complex technology and make it accessible to anyone.

Blynk is designed for IOT, and is focused on marketing products for individuals, companies, world-class users and IT professionals. This application can control hardware from a distance, can display sensor data, can store data, visualize and do many other things. Blynk itself has features including:

i. Connection using USB, Ethernet, WiFi, Bluetooth.

ii. Easy to use collection of views.

iii. Direct pin modification without writing code.

iv. Send notifications via Twitter and email.

v. Having a server as a provider of data communication networks between smartphones and hardware, especially micron controllers.

vi. Has a library that is useful for all hardware platforms for communication with servers and processing all incoming and outgoing commands. 


\subsection{Block diagram}

The fuel volume monitoring system in this tool starts from changes in the voltage value of the fuel meter driver due to the position of the ups and downs of the buoys from the motorcycle gas tank. The data is in the form of analog voltage quantities which are input to the analog pin A0 on the NodeMCU microcontroller. The analog voltage value that goes into the ADC is changed to digital first, and the process occurs inside the microcontroller. In the tire pressure monitoring system, a pressure sensor is connected to the A0 foot on the ADS1115 ADC. The work process that occurs on the external ADS1115 is similar to the internal ADC microcontroller NodeMCU, that is by converting analog voltage into digital values that will be read on the microcontroller. The microcontroller will receive digital data that has undergone a conversion from analog voltage both from the internal ADC NodeMCU and the external ADC 1115.

The process in the Figure 4 diagram indicates that an Android smartphone is connected to the Blynk server wirelessly as a monitor of BBM availability and tire pressure. The microcontroller with the ESP8266 WiFi module serves as a bridge between the Blynk server and the Android smartphone, and the position of the microcontroller must always be connected to the internet. The Blynk application has a security system when logging in with the method must first type using an email and password (password) to avoid abuse from other users. The microcontroller that acts as the brain of the entire system is connected to a WiFi network that is connected to the internet can access the database server and can be opened via a smartphone.

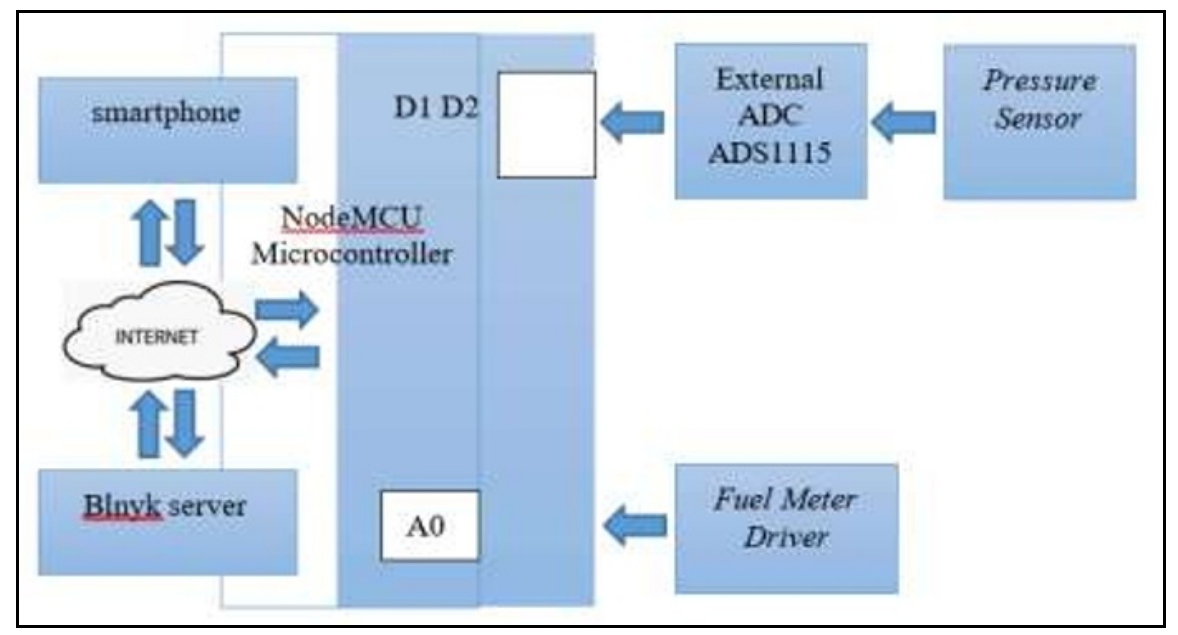

Fig. 4. Block diagram.

\section{Conclusion and result}

From the results of the design, manufacture, testing, and measurements that have been made, the conclusions can be taken as follows: (i) The tool functions in accordance with the design goals, namely the tool can monitor the volume of gasoline and tire pressure using the IOT concept with a smartphone, making it easier for users to monitor their value in the form of available volumes, gasoline prices, kilometers traveled the value of tire pressure when conditions are problematic or normal; (ii) The value of the fuel meter voltage and the pressure sensor are directly proportional to the amount of gas volume and the tire pressure value. If the gasoline volume and tire pressure are reduced, the fuel meter voltage and 
pressure sensor decrease. Meanwhile, if the gasoline volume and tire pressure are added, the fuel meter voltage and pressure sensor increase; (iii) Monitoring by using IOT is not limited by regional coverage, but the tool must be connected to the internet network so that it can still send and receive data through Blynk; (iv) Constraints of testing system range are the strength of cellular signals used by users experiencing fluctuations which means they can weaken and strengthen, but do not cause damage to the tool.

\section{References}

1. A. Meola. The Internet of things. [Online] from https://www.businessinsider.com/ internet-of-things-definition?IR=T (2018). [Accessed on 1 June 2018].

2. D. Minoli. Building the internet of things with IPv6 and MIPv6. New Jersey: John Wiley \& Sons, Inc. (2013). p. 392.

https://onlinelibrary.wiley.com/doi/book/10.1002/9781118647059

3. S. Gaglio. Advances in intelligent systems and computing. New York: Springer (2014). p. 325. https://link.springer.com/book/10.1007/978-3-319-45991-2

4. P. Waher. Learning internet of things. Birmingham: Packt Publishing Ltd. (2015). p. 242. https://www.amazon.com/Learning-Internet-Things-Peter-Waher/dp/1783553537

5. A. McEwen, H. Cassimally. Defining the internet of things. New Jersey: Wiley (2016). p. 336. https://www.amazon.com/Designing-Internet-Things-AdrianMcEwen/dp/ $111843062 \mathrm{X}$

6. NodeMCU. NodeMCU v3. [Online] from https://www.nodemcu.com/index_en.html (2018). [Accessed on 23 June 2019].

7. Nice. How fuel gauge works [Online] from https://auto.howstuffworks.com/fuel-gauge.htm (2017). [Accessed on 21 June 2019].

8. TE Connectivity. Pressure sensor. [Online] from https://www.te.com/usa-en/products/ sensors/pressure-sensors.html?source=header-match (2017). [Accessed on 27 May 2019].

9. K. Karvinen, T. Karvinen. Getting started with sensors: Measure the world with electronics, arduino, and raspberry pi. Canada: Maker Media, Inc. (2014). https://www.amazon.com/Getting-Started-Sensors-ElectronicsRaspberry/dp/1449367089

10. A. Guerrieri, V. Loscri, A. Rovella, G. Fortino. Management of cyber physical objects in the future internet of things: Methods, architectures and applications. New York: Springer International Publishing (2016). p. 174. https://www.springer.com/gp/book/9783319268675

11. Adafruit. ADS1115 16-Bit ADC-4 channel with Programmable Gain Amplifier [Online] from https://www.adafruit.com/product/1085 (n.d.). [Accessed on 2 June 2019]. 